\title{
Ocean temperature and salinity inverted from combined hydrographic and seismic data
}

\author{
C. Papenberg, ${ }^{1}$ D. Klaeschen, ${ }^{1}$ G. Krahmann, ${ }^{1}$ and R. W. Hobbs ${ }^{2}$ \\ Received 10 December 2009; revised 13 January 2010; accepted 19 January 2010; published 16 February 2010.
}

[1] Recently seismic reflection methods have been successfully applied to oceanographic issues. Here, we present a new approach, combining XBT and CTD surveys with seismic observations, to visualize long sections with a resolution down to a few meters. The challenge to a full investigation of mixing processes has been the tremendous span of spatial scales ranging from hundreds of kilometers to centimeters. Traditional hydrographic observations could only resolve the large scale effects by measuring temperature and salinity profiles at discrete locations typically several kilometers apart, whereas dedicated localized measurements allowed investigation of the ocean fine structure at the other end of the spatial spectrum. The intermediate scales have in contrast been difficult to observe systematically. Here we present temperature and salinity data inverted from seismic observations that cover the intermediate scales and provide a new approach to image mesoscale processes and allow the investigation of their dynamics at unprecedented resolution. Citation: Papenberg, C., D. Klaeschen, G. Krahmann, and R. W. Hobbs (2010), Ocean temperature and salinity inverted from combined hydrographic and seismic data, Geophys. Res. Lett., 37, L04601, doi:10.1029/2009GL042115.

\section{Introduction}

[2] Marine seismic reflection profiling has been used over the past 40 years to image the interior of the earth [Lines, 2005]. In marine seismic experiments an acoustic pulse, a shot, is generated typically every 10 to $20 \mathrm{~s}$ using compressed air. This pulse is abruptly released a few meters below the sea surface, using a system of 'air-guns' towed behind a vessel. The generated compressional wave propagates down through the water and the subsurface. Each time the wave encounters a change in the acoustic impedance, a fraction of the acoustic energy is reflected. The impedance, for acoustic media like water, is defined as the product of compressional sound speed and density within a given layer. The upward propagating reflected wave-field is detected on an array of hydrophones also towed by the vessel. As the vessel moves through the water, the repeated shots create a section of impedance boundaries within the water column. The relative amplitude of the reflected signals is a function of the reflection coefficient, which in turn is related to the impedance contrast across the boundary [Aki and Richards, 1980]. Here we use this profiling method to ensonify the water column and to map its boundaries. However, unlike

\footnotetext{
${ }^{1}$ Leibniz Institute of Marine Sciences at University of Kiel (IFMGEOMAR), Kiel, Germany.

${ }^{2}$ Earth Sciences Department, Durham University, Durham, UK.
}

Copyright 2010 by the American Geophysical Union. 0094-8276/10/2009GL042115\$05.00 the solid earth, where the boundaries between two rock masses may be sharp and a description by the impedance above and below the boundary suffice, the boundaries in the ocean are affected by diffusion processes, so that the reflection coefficient needs to be described by continuous profiles of the vertical impedance gradient or the underlying properties sound speed and density [Ruddick et al., 2003]. These quantities can be related to temperature and salinity of the water through the equation of state [Millero et al., 1980].

[3] Previous studies in seismic oceanography have used the method to image water mass boundaries [Holbrook et al., 2003; Nandi et al., 2004; Nakamura et al., 2006] or directly analyzed the seismic reflection data for the shape of the reflection boundaries [Krahmann et al., 2008; Holbrook and Fer, 2005], but only a few have focused on inverting ocean temperature and salinity from seismic data directly as often the uncertainties of the inverted properties have been deemed too high in comparison with XBT (Expendable Bathythermograph) and CTD (Conductivity-TemperatureDepth) observations. Páramo and Holbrook [2005] calculated temperature contrasts for isolated seismic reflectors and Wood [Wood et al., 2008] presented results of a 1-D full-waveform inversion applied to ocean seismic reflection data though they assumed constant salinity. These studies, although restricted to $1-\mathrm{D}$, show the potential of inverting seismic data to obtain physical oceanographic parameters. However to potentially address the questions of mesoscale interactions, and e.g. dissipation rates, we need to invert full seismic sections covering tens to hundreds of kilometers.

\section{Method}

[4] The fundamental step in our seismic analysis is the careful pre- and post-processing of multi-channel seismic data and preservation of its signal amplitudes, thereby allowing us to generate a true-amplitude time-migrated seismic section. The following deconvolution uses the seismic source signal, to compute the amplitude reflection coefficients. These are then inverted for sound speed assuming a depth dependent density profile (Figures 1a and 1d). In the final stage of the inversion we convert sound speed into temperature and salinity. For this we use a T-S(z) relationship (temperature-salinity) (Figure 1b) derived from CTD observations and the equation of state for seawater [Millero et al., 1980] to simultaneously calculate temperature and salinity for the whole profile in an iterative process (Figure 1a).

[5] One key element of the process is to derive sound speed from the seismic data.

[6] We derive sound speed changes from reflection amplitudes which in turn requires amplitude preserving preprocessing steps. These include the removal of the direct wave, bandpass filtering, angle and frequency dependent 

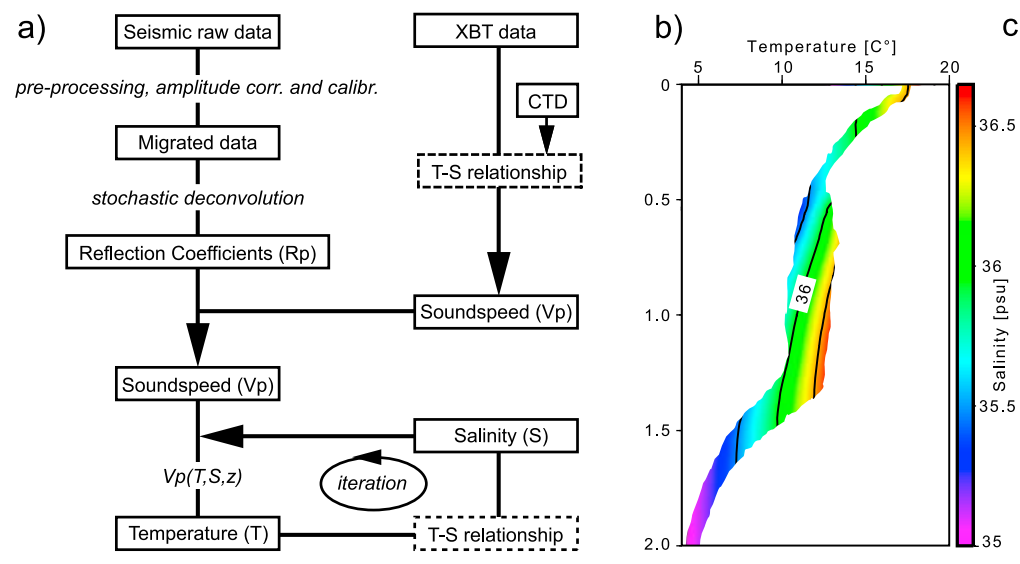

c)

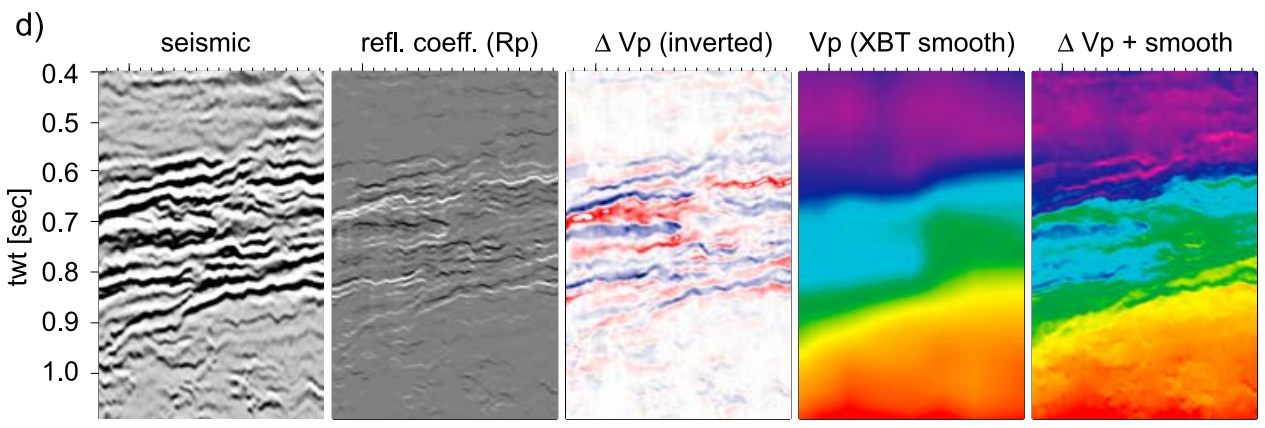

Figure 1. (a) Flow chart of the inversion steps applied to seismic and hydrographic (XBT,CTD) data to invert temperature and salinity. The inversion is divided in two stages: 1 . Seismic processing to invert for sound speed and 2 . the conversion of sound speed to temperature and salinity in an iterative process. (b) Displayed is the depth dependent temperature to salinity conversion chart derived from linear regressions of the CTD measurements. (c) The quality of the linear regression is shown by the $\mathrm{R}^{2}$ values. Lower values of $\mathrm{R}^{2}$ occur only at depths where there is little total variance in $\mathrm{T}$ and $\mathrm{S}$. (d) image panels, displayed in two-way-traveltime (twt), to sketch the steps from the seismic data to the inverted sound speed, from left to right: seismic migrated data, deconvolution result providing the reflection coefficients (Rp), Rp converted to sound speed (D Vp) after being low-cut filtered providing relative sound speed perturbation, background absolute sound speed model, derived from XBTs, and final sound speed (Vp) model after merging background and relative sound speed.

receiver/source directivity corrections, and compensation for spherical divergence. The amplitudes were calibrated, using the primary and multiple reflections of the seafloor at a horizontally layered segment. The next step is the seismic migration, which increases the lateral and temporal resolution by reducing the Fresnel zone and correctly images the subsurface structures in space and depth with amplitudes corresponding to the impedance contrast in the subsurface. Unlike in 3-D elastic subsurface media, where sound speed variations can be abrupt and complex, here in the acoustic media of the ocean, we expect only smooth lateral sound speed changes and small reflector dips $\left(<5^{\circ}\right)$. The most efficient approach is therefore a true amplitude pre-stack time migration, which analytically calculates travel time and weight functions to recover the amplitude information, the key parameter in our processing sequence, to derive the reflection coefficients. Migration velocities used in this step were derived from simultaneously measured XBT data, converted to sound speed. The migrated data now represents true reflection coefficients still convolved with the source signal (wavelet). A deconvolution process is needed to remove the wavelet [Oldenburg et al., 1983]. The stochastic deconvolution method applied here simultaneously estimates a wavelet and the reflection signal. It is based on the convolution model, assuming sparse vertical sequences, a wavelet common to all traces, and a continuous reflectivity in the horizontal direction. The algorithm recovers a nonminimum phase wavelet and closely spaced reflectors using a least square method. The process starts with an initial guess of the reflectivity and uses the seismic data to find a wavelet estimate. In the next step it assumes the wavelet to be correct and estimates a new reflectivity. This reflectivity improves upon the initial guess. The procedure then improves the wavelet and reflectivity until the process converges. The deconvolved data now represents a section of reflection coefficients, which are then converted to sound speed. We recursively calculated sound speed from top to bottom for a given density profile starting with a known sound speed at the sea-surface. Due to the band-limited nature of the seismic signal $(>6 \mathrm{~Hz})$, the long vertical wavelength $(>250 \mathrm{~m})$ variation of absolute sound speed values cannot be recovered from the seismic data. Instead, we used a smooth background sound speed model (vertical wavelength $>250 \mathrm{~m}$ ), derived from XBTs, recorded every $3 \mathrm{~km}$ along the transect. XBTs only record temperature, so to calculate the sound speed [Millero et al., 1980] we apply a T-S(z) relationship from data acquired by a second vessel, which conducted CTD casts throughout the entire survey. These CTD casts showed no seismically significant density variations, and therefore support our assumption that the 


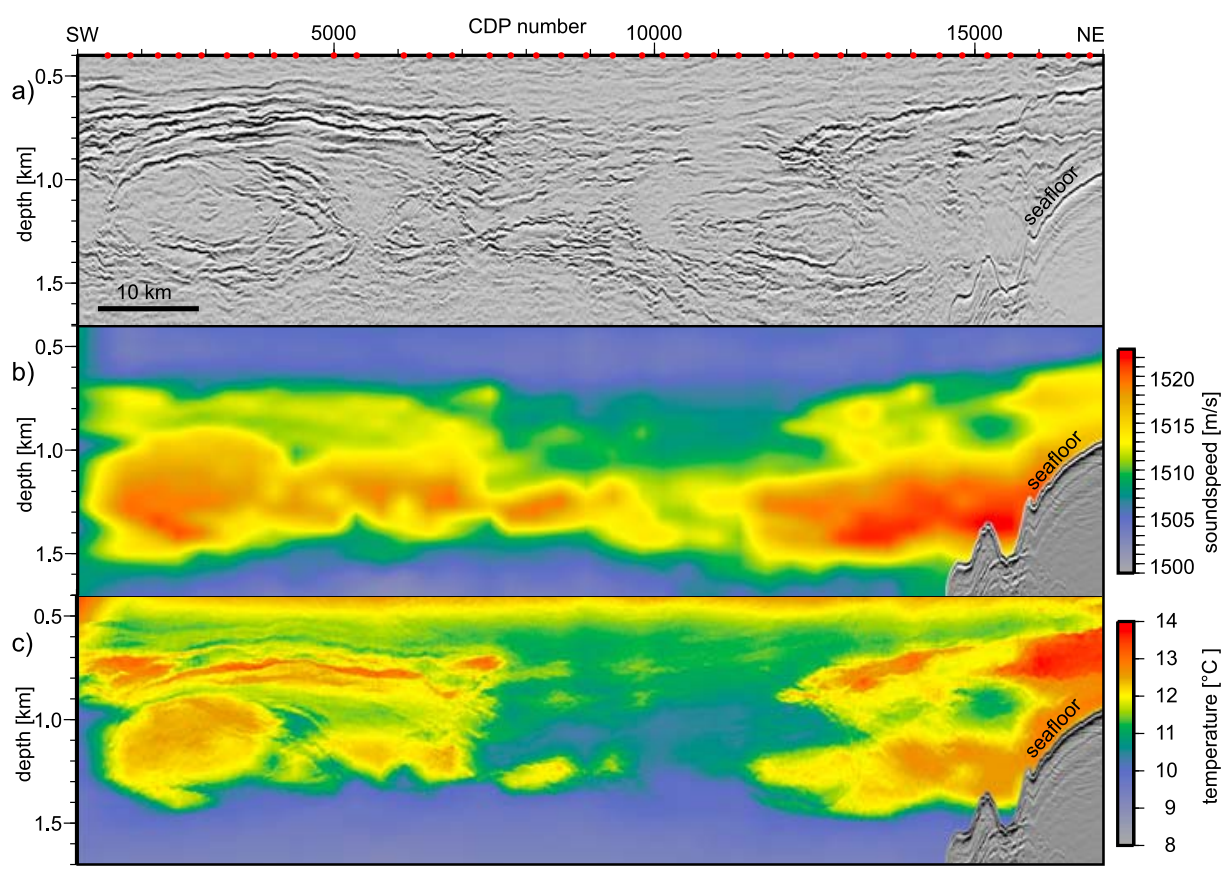

Figure 2. (a) Seismic reflection image of the main profile cutting the main vein of the Mediterranean Outflow Water in the Northeast and imaging a Meddy in the Southeast part of the profile. The section is converted to depth using the background sound speed model. The seafloor visible in the eastern part of the profile is scaled down and adjusted to the reflectance of the water column. (b) XBT-derived sound speed background model generated from simultaneous XBT casts (red circles on top axis). (c) Temperature model, after the inversion process using combined seismic and hydrographic data, now providing a higher resolution and imaging fine structures of the dynamic oceanic features.

amplitude of the reflections is mainly determined by the short wavelength variation in sound speed. The final sound speed model is the summation of the smooth background model and the inverted short wavelength model.

[7] The T-S(z) relationship used in the previous step is now used to compute temperature and salinity from the inverted sound speed model in an iterative process. For the first iteration we use a constant salinity of $36 \mathrm{psu}$ and derive a temperature only variation to match the computed sound speed. For the next iterations the salinity is adjusted according to the $\mathrm{T}-\mathrm{S}(\mathrm{z})$ relationship using the temperature from the previous iteration until error is less than the noise in the data. This approach works because sound speed is dominated by the effect of temperature and only weakly affected by salinity with sensitivities that ensure convergence, and because in the region of interest the mixing of two main water masses creates, at each depth, a single linear mixing curve thereby leading to a unique pair of temperature and salinity for a given sound speed. Employing the steps outlined above enables the visualisation of fine layers in the temperature (Figures 2c and 3a) and salinity (Figure 3b) domains that are directly assessable to physical oceanography analysis over the full range of mesoscale length-scales in the horizontal direction.

\section{Results}

[8] We applied this method to multi-channel seismic data acquired in the Gulf of Cadiz during the GO cruise [Hobbs et al., 2007], a region known for the generation of mesoscale Meddies [e.g., Armi et al., 1989; Serra et al., 2005]. Meddies are rotating eddies of Mediterranean Outflow Water formed from the warm saline water flowing out of the Straits of Gibraltar and along the southern margin of Iberia [Ambar et al., 2008]. The Portimao Canyon, south of Portugal, disrupts this flow and is a locus for the creation of Meddies which detach from the main vein and drift away from the continental slope at the depth of neutral buoyancy (between $600-1400 \mathrm{~m}$ ). Where the warm saline Mediterranean water and the cold Atlantic water mix, they generate strong acoustic impedance contrasts, and consequently create clear reflections that image the mixing processes (Figure 2a). Simultaneously measured temperature profiles (XBTs, represented by red circles on top axis in Figure 2a) were converted to a background sound speed model (Figure 2b) and already visualize regions of relatively higher sound speeds, induced by the warm and saline Mediterranean water. After we have used the seismic data to invert for temperature (Figure 2c) and salinity the details of the mixing become much more visible. A Meddy with its main core between 800 and $1400 \mathrm{~m}$ can be clearly identified in the western part of the profile, while the eastern part cuts the main vein of Mediterranean Outflow Water. This Meddy was crossed a few days later on another seismic profile and the inverted temperature and salinity models (Figures $3 \mathrm{a}$ and $3 \mathrm{~b})$ enhance the structural image of the Meddy, especially the fine filaments at the top of the main core.

[9] To evaluate our results, we compared in-situ XBT temperature and XBT-derived salinity profiles with the inverted values. Our temperature results can be independently verified (Figure 4a) and match the XBT data within an average error of $0.1^{\circ} \mathrm{C}$. In Figure 4 these correlations are displayed at three locations, marked in Figure $3 \mathrm{a}$ as dotted lines. The CTD measurements were temporally not coinci- 


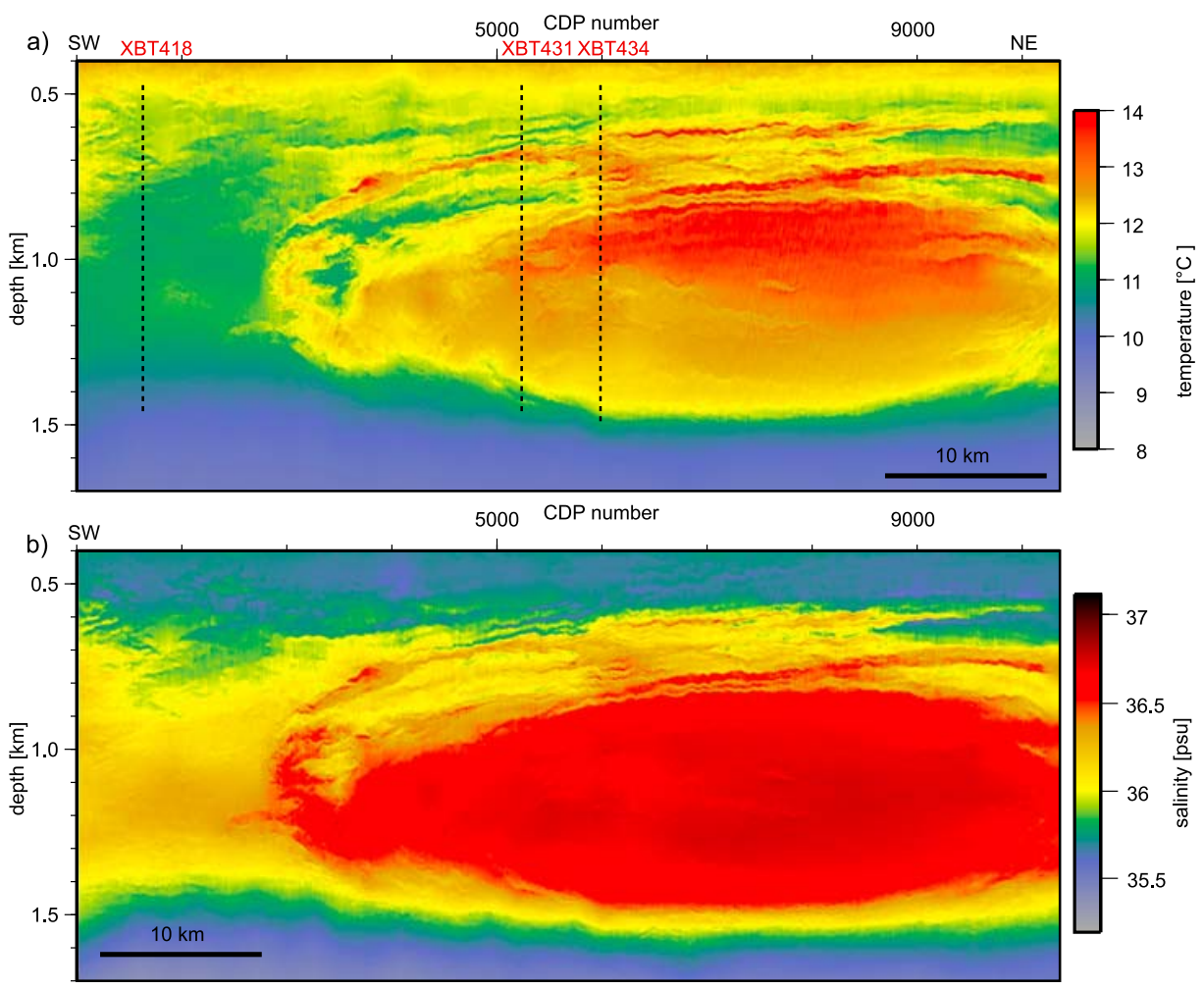

Figure 3. (a) Temperature and (b) salinity model after the inversion process using combined seismic and hydrographic data. The model provides a detailed image of fine structures in and around the Meddy, especially the fine filaments on top of the Meddy.

dent with the seismic traces and therefore salinity could not be independently evaluated. However, the inverted salinity values fit to the XBT-derived salinity within an error of
0.1 psu (Figure 4b). Although we do not have coincidental salinity data, our fit does reflect the robustness of our inversion. A potential uncertainty of our inversion is the non-
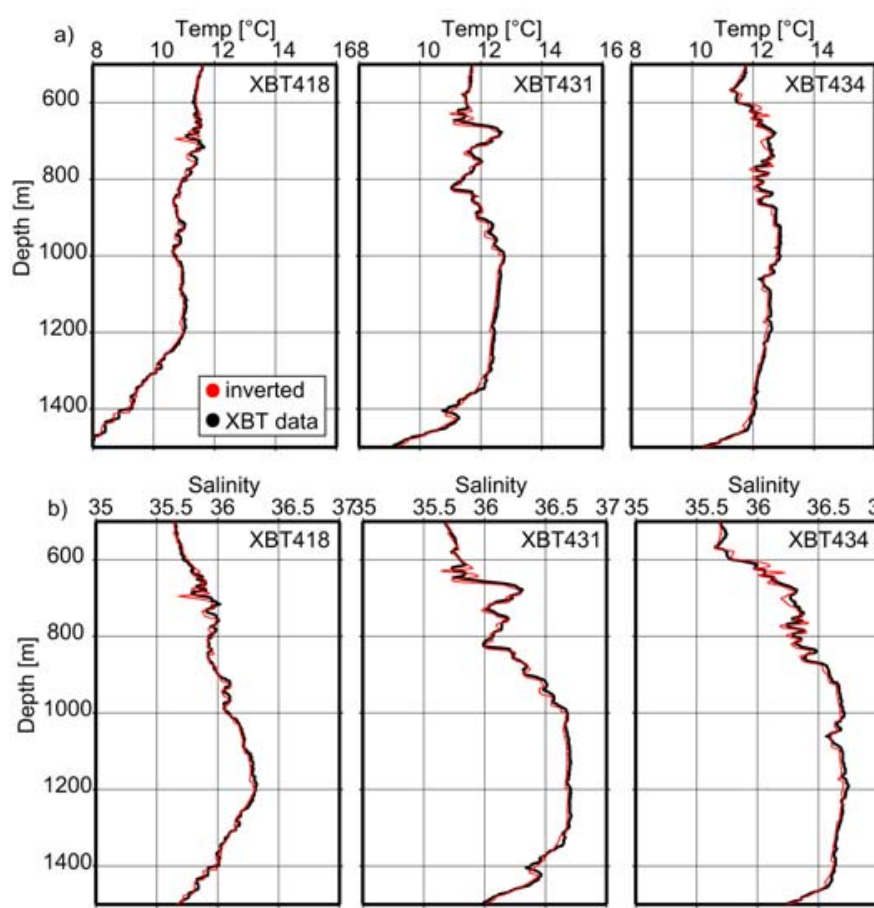

Salinity
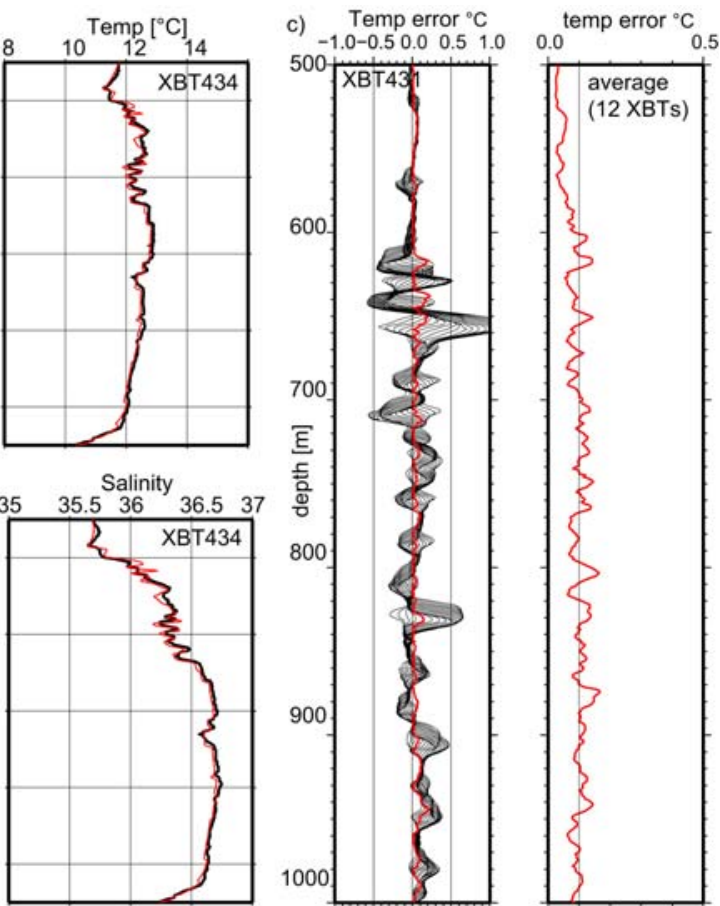

Figure 4. Error estimation of inverted (red) (a) temperature and (b) salinity vs. coincident XBT profiles (black). Depth error of 10 meters was allowed to correlate temperature and salinity values. Minimum deviation was taken for error estimates. (c) Mean error over 12 XBTs along profile GO-LR-12, displayed in Figure 3. 
uniqueness of the deconvolution process at small scales $(<15 \mathrm{~m}$, equivalent to $1 / 4$ th of the wavelength of the dominant frequency of the source wavelet). A more critical aspect is the validity of the T-S relationship, which in this study is averaged for the entire survey area. The CTD data do, however, show that in this region for a given depth a linear $\mathrm{T}-\mathrm{S}$ relationship is either a particularly good fit or there is little temperature variance (Figure 1c). Recently, Sallarès [Sallarès et al., 2009] and colleagues investigated the influence of salinity and temperature on seismic reflection amplitudes in the same area and concluded, that the main contribution to seismic reflectivity is that of sound speed variations (90-95\%), relative to density (5-10\%). Likewise, temperature contrasts account on average for $\sim 80 \%$ of the reflectivity, and salinity for the remaining $\sim 20 \%$. However, the partial contribution of the different properties is highly variable for different localities. Sallarès observations are reflected in the relatively large discrepancies (bigger than $0.1^{\circ} \mathrm{C}$ and $0.1 \mathrm{psu}$, respectively) observed in Figures $4 \mathrm{a}$ and $4 \mathrm{~b}$ at a depth of 700 meters, which marks the top of the Meddy. We assign this region to oceanic layers, where the density and T-S relationship are more complex than assumed in the inversion process. These uncertainties could possibly be minimized by applying a regionally varying T-S relationship, which in turn would need a denser grid of CTD measurements than available here. Another aspect is an observed depth error when correlating measured and inverted values. We have observed discrepancies of up to $10 \mathrm{~m}$ in depth between XBT and seismic data. We address this error to lateral mis-positioning of XBTs or to uncertainties within the depth conversion of the XBT data [Kizu et al., 2005], which is based on a fall rate equation provided by the XBT manufacturer. The influence of sound speed can be neglected because depth is not sensitive to the vertical sound speed variations $(<5 \mathrm{~m} / \mathrm{s})$ found here. To estimate the error of our inversion we accounted for this problem and allowed a depth error of $10 \mathrm{~m}$ when finding the best correlation between measured and inverted values (Figure 4c).

[10] Another influence on the reflection amplitude is the non-stationary aspect of moving reflectors and the applied sound speed model to stack the data [Fortin and Holbrook, 2009]. Both effects are linked to each other during the processing phase, because moving reflectors generate apparent dips in the seismic shot record [Klaeschen et al., 2009]. Standard seismic processing and acquisition methods were developed to study the subsurface and assume stationary targets during the time of acquisition. In the ocean, water masses are in motion, and if the movement velocity (e.g. currents) is fast, the 'stationary'assumption is violated and consequently the reflection amplitude is weaker. During the inversion, this can lead to an underestimation of the reflection coefficient and subsequently to smaller temperature and salinity values. Peak currents were, however, simultaneously measured in our survey area and do not exceed $0.4 \mathrm{~m} / \mathrm{s}$. Thus they have only minor influence on the inversion process presented here and are expressed within the general uncertainty.

\section{Conclusion}

[11] One major benefit of seismic oceanography is the dense horizontal sampling of acoustic reflection images. With seismic oceanography we can thus visualize water mass boundaries over long sections (of tens to a hundred kilometers) with a resolution down to a few meters. We can take advantage of the dense sampling provided by seismic reflection methods and have successfully derived temperature and salinity sections from combined seismic and hydrographic data. Seismic profiles over several tens of kilometers can be inverted based on a representative T-S relationship. The accuracy of the inversion result hereby is dependent on the complexity of the oceanic regime and subsequently sensitive to the T-S relationship. We inverted temperature and salinity from combined data acquired in a strongly dynamic region, known for the generation of mesoscale Meddies, with an accuracy of $0.1^{\circ} \mathrm{C}$ and $0.1 \mathrm{psu}$, respectively. The inverted data provides a new approach to image mesoscale oceanic processes and allows us to study its dynamics at unprecedented resolution.

[12] Acknowledgments. This work was supported by the EU project GO(15603) (NEST) and DFG (KR 3488/1-1). The authors thank the officers and crew on the RRS Discovery and FS Poseidon for their professional assistance during the GO experiment. Seismic sections were produced using SEISMOS and OMEGA/Western-Schlumberger, and Seismic Unix software, visualization used GMT.

\section{References}

Aki, K., and P. G. Richards (1980), Quantitative Seismology: Theory and Methods, W. H. Freeman, San Francisco, Calif.

Ambar, I., N. Serra, F. Neves, and T. Ferreira (2008), Observations of the Mediterranean Undercurrent and eddies in the Gulf of Cadiz during 2001, J. Mar. Syst., 71, 195-220, doi:10.1016/j.jmarsys.2007.07.003.

Armi, L., et al. (1989), Two years in the life of a Mediterranean salt lens, J. Phys. Oceanogr., 19, 354-370, doi:10.1175/1520-0485(1989) 019<0354:TYITLO >2.0.CO;2.

Fortin, W. F. J., and W. S. Holbrook (2009), Sound speed requirements for optimal imaging of seismic oceanography data, Geophys. Res. Lett., 36, L00D01, doi:10.1029/2009GL038991.

Hobbs, R., et al. (2007), GO - Geophysical Oceanography: A new tool to understand the thermal structure and dynamics of oceans, D318 Cruise Rep., Durham Univ., Durham, U. K. (Available at http://www.dur.ac. uk/eu.go/cruise/report.html)

Holbrook, W. S., and I. Fer (2005), Ocean internal wave spectra inferred from seismic reflection transects, Geophys. Res. Lett., 32, L15604, doi: $10.1029 / 2005$ GL023733.

Holbrook, W. S., P. Páramo, S. Pearse, and R. W. Schmitt (2003), Thermohaline fine structure in an oceanographic front from seismic reflection profiling, Science, 301, 821-824, doi:10.1126/science.1085116.

Kizu, S., S. Ito, and T. Watanabe (2005), Inter-manufacturer difference and temperature dependency of the fall-rate of T- 5 expendable bathythermograph, J. Oceanogr., 61, 905-912.

Klaeschen, D., R. W. Hobbs, G. Krahmann, C. Papenberg, and E. Vsemirnova (2009), Estimating movement of reflectors in the water column using seismic oceanography, Geophys. Res. Lett., 36, L00D03, doi:10.1029/ 2009GL038973.

Krahmann, G., P. Brandt, D. Klaeschen, and T. Reston (2008), Middepth internal wave energy off the Iberian Peninsula estimated from seismic reflection data, J. Geophys. Res., 113, C12016, doi:10.1029/ 2007JC004678.

Lines, L. (2005), Addressing Milo's challenges with 25 years of seismic advances, Leading Edge, S24, 32-34, doi:10.1190/1.2112389.

Millero, F. J., C. T. Chen, A. Bradshaw, and K. Schleicher (1980), A new high pressure equation of state for seawater, Deep Sea Res., Part A, 27, 255-264, doi:10.1016/0198-0149(80)90016-3.

Nakamura, Y., T. Noguchi, T. Tsuji, S. Itoh, H. Niino, and T. Matsuoka (2006), Simultaneous seismic reflection and physical oceanographic observations of oceanic fine structure in the Kuroshio extension front, Geophys. Res. Lett., 33, L23605, doi:10.1029/2006GL027437.

Nandi, P., W. S. Holbrook, S. Pearse, P. Páramo, and R. W. Schmitt (2004), Seismic reflection imaging of water mass boundaries in the Norwegian Sea, Geophys. Res. Lett., 31, L23311, doi:10.1029/2004GL021325.

Oldenburg, D. W., T. Scheuer, and L. L. Levy (1983), Recovery of acoustic impedance from reflection seismograms, Geophysics, 48(10), 1318-1337, doi:10.1190/1.1441413. 
Páramo, P., and W. S. Holbrook (2005), Temperature contrasts in the water column inferred from amplitude-versus-offset analysis of acoustic reflections, Geophys. Res. Lett., 32, L24611, doi:10.1029/2005GL024533.

Ruddick, B., H. Song, C. Dong, and L. Pinheiro (2009), Water column seismic images as maps of temperature gradient, Oceanography, 22 (1), 192-205.

Sallarès, V., B. Biescas, G. Buffett, R. Carbonell, J. J. Dañobeitia, and J. L. Pelegrí (2009), Relative contribution of temperature and salinity to ocean acoustic reflectivity, Geophys. Res. Lett., 36, L00D06, doi:10.1029/2009GL040187.

Serra, N., I. Ambar, and R. H. Käse (2005), Observations and numerical modelling of the Mediterranean outflow splitting and eddy generation, Deep Sea Res., Part II, 52, 383-408, doi:10.1016/j.dsr2.2004.05.025.
Wood, W. T., W. S. Holbrook, M. K. Sen, and P. L. Stoffa (2008), Full waveform inversion of reflection seismic data for ocean temperature profiles, Geophys. Res. Lett., 35, L04608, doi:10.1029/2007GL032359.

R. W. Hobbs, Earth Sciences Department, Durham University, Durham, DH1 3LE, UK

D. Klaeschen, G. Krahmann, and C. Papenberg, Leibniz Institute of Marine Sciences at University of Kiel (IFM-GEOMAR), Kiel D-24148, Germany. (cpapenberg@ifm-geomar.de) 